\title{
Title: Health-related quality of life after open transhiatal and transthoracic surgery for oesophageal cancer
}

Authors: Joonas H Kauppila ${ }^{1,2}$, Asif Johar ${ }^{1}$, James A Gossage ${ }^{3,4}$, Andrew R Davies ${ }^{3,4}$, Janine Zylstra $^{3,4}$, Jesper Lagergren ${ }^{1,3,4}$, Pernilla Lagergren ${ }^{1}$

Affiliations: ${ }^{1}$ Upper Gastrointestinal Surgery, Department of Molecular medicine and Surgery, Karolinska Institutet, Karolinska University Hospital, 17176 Stockholm, Sweden, ${ }^{2}$ Cancer and Translational Medicine Research Unit, Medical Research Center, University of Oulu and Oulu University Hospital, Oulu, Finland, ${ }^{3}$ Division of Cancer Studies, King's College London and ${ }^{4}$ Department of Surgery, Guy's and St Thomas' Oesophago-Gastric Centre, London, England.

\section{Author correspondence to:}

Joonas H Kauppila, Upper Gastrointestinal Surgery, Department of Molecular medicine and Surgery, Karolinska Institutet, Karolinska University Hospital, 17176 Stockholm, Sweden Email: joonas.kauppila@ki.se, Tel. +46 8-517 709 83, Fax: +46 8-517 76280

Sources of funding: Funding was provided by Guy's and St Thomas' Charity, London, United Kingdom. The study sponsors had no role in the design of the study, data collection, analysis or interpretation of the results, the writing of the manuscript or the decision to submit the manuscript for publication. The authors declare no conflicts of interest.

Manuscript category: Original article 


\section{Abstract}

Background: Transhiatal and transthoracic approaches for oesophagectomy in patients with oesophageal cancer have similar survival rates, but whether these approaches differ regarding health-related quality of life (HRQOL) is uncertain and was examined in this study.

Methods: Patients undergoing transhiatal or transthoracic surgery for lower third oesophageal or gastro-oesophageal junctional cancer were prospectively recruited in a cohort study from St Thomas' Hospital, London, United Kingdom, in 2011-2015, with follow-up until 2016. HRQOL outcomes were measured at 6 and 12 months after surgery using validated written questionnaires (EORTC QLQ-C30 and QLQ-OG25). Linear mixed models provided mean score differences (MSD) with 95\% confidence intervals (CI), adjusted for preoperative HRQOL, age, physical status (ASA-grade), tumour location, tumour stage, neoadjuvant therapy, adjuvant therapy and postoperative complications. MSDs $\geq 10$ were regarded clinically relevant and thus tested for statistical significance using T-test.

Results: Among 146 eligible patients undergoing transhiatal $(n=86,58.2 \%)$ or transthoracic oesophagectomy ( $n=60,41.8 \%), 111(78.7 \%$ of the alive patients) answered the HRQOL questionnaires at 6 months and 76 (72.4\% of the alive patients) at 12 months. At 6 months, transthoracic oesophagectomy was associated with worse role function (MSD -13, 95\% CI $25-0, p=0.046)$. At 12 months, patients in transthoracic group had more nausea and vomiting

(MSD 11, 95\% CI 0-22, $p=0.045$ ) dyspnoea (MSD 13, 95\% CI 1-25, $p=0.029$ ) and constipation (MSD 20, 95\% CI 7 to 33, $p=0.003$ ) compared to the transhiatal group.

Conclusions: Transhiatal oesophagectomy seems to offer better HRQOL compared to transthoracic oesophagectomy 6 and 12 months after surgery.

Keywords: Oesophagus; neoplasm; survivorship; HRQOL. 


\section{Introduction}

The curative treatment of most patients diagnosed with cancer of the oesophagus or the gastro-oesophageal junction includes oesophagectomy, ${ }^{1,2}$ but the optimal surgical approach remains uncertain. ${ }^{3}$ The two predominant open surgical approaches for oesophageal cancer are the transhiatal and transthoracic oesophagectomy. ${ }^{3}$ In transhiatal approach, oesophagectomy is conducted by accessing the distal oesophagus from abdominal incision and through the diaphragm. ${ }^{4}$ Oesophago-gastric anastomosis is then done through a left-side incision in the neck. ${ }^{4}$ Transthoracic oesophagectomy is conducted through, often separate, incisions in the abdomen and the thorax. Anastomosis is usually done intrathoracally. ${ }^{3}$ The transhiatal and transthoracic approaches seem to provide similar oncological outcomes, ${ }^{3,5-7}$ which has also been reported from our centre. ${ }^{8}$

Severe deterioration in HRQOL is a major concern in most oesophageal cancer patients during the first postoperative year, and sometimes longer. ${ }^{9,10}$ Comorbidities and postoperative complications are associated with poor recovery in HRQOL. ${ }^{11,} 12$ Therefore, transhiatal oesophagectomy is sometimes preferred in frail patients, for example those of older age or with pulmonary comorbidities. ${ }^{13}$ It is less clear whether these two procedures differ regarding postoperative health-related quality of life (HRQOL) outcomes because there is only one previous study on the topic that suggests favourable outcome after transhiatal oesophagectomy up to 3 months after surgery. ${ }^{14}$ As thoracotomy is not used in the transhiatal approach, ${ }^{4}$ it was hypothesised that transhiatal approach causes less surgical trauma compared to transthoracic oesophagectomy, and might reduce pain and pulmonary morbidity, ${ }^{7}$ and thus result in better postoperative HRQOL.

The aim of this prospective cohort study was to test the hypothesis that transhiatal oesophagectomy for cancer is associated with better HRQOL outcomes at 6 and 12 months postoperatively, compared to transthoracic oesophagectomy. 


\section{Methods}

\section{Study design}

This was a single-centre prospective cohort study from St Thomas' Hospital in London, United Kingdom during the period November 2011 to February 2016. The open transhiatal approach was compared with the open transthoracic approach with regards to HRQOL outcomes at 6 and 12 months after surgery. Informed consent was obtained from all study participants. The study was approved by the National Research Ethics Services in London and West Midlands (REC references 11/LO/0335 and 13/WM/0131).

\section{Clinical management}

All patients were managed by a well-established upper gastrointestinal cancer multidisciplinary team. Study patients had a standard protocol of investigation, including oesophagogastroduodenoscopy and computed tomography. Additional diagnostic measures, such as endoscopic ultrasonography, fluorodeoxyglucose positron emission tomography or laparoscopy were conducted when clinically indicated. Indications and regimens for neoadjuvant therapy followed established evidence-based standards. ${ }^{15}$ The neoadjuvant regimen information was available in $119(93.0 \%)$ of the 128 patients undergoing neoadjuvant therapy. Of these, $115(96.6 \%)$ had a platinum-based triple therapy and four (3.4\%) had capecitabine with or without platinum-based agents or antibodies.

\section{Data collection}

The prospectively collected clinical information included patient and tumour characteristics, surgical details and other treatment variables, pre-defined complications occurring within 30 days of surgery, and written HRQOL questionnaire responses collected at baseline (preoperatively), and at 6 and 12 months after surgery. The American Society of 
Anesthesiologists (ASA) physical status (scale 1 to 5) was used to assess the fitness of the patients at the time of surgery. The precise tumour location and stage were defined on the basis of pathological examination of the resected tumour specimen according to the 7 th edition of the Union for International Cancer Control (UICC) and the American Joint Committee on Cancer (AJCC) Cancer Staging Manual. ${ }^{16}$ Post-operative complications were recorded using the well-validated Clavien-Dindo 'Classification of Surgical Complications' grades. $^{17}$

\section{Surgical approach (exposure)}

Decisions on surgical approach for the individual patients were made in the multidisciplinary meetings. The transhiatal approach was used in older or frail patients with early tumours at the gastro-oesophageal junction. Transhiatal oesophagectomy was conducted through laparotomy (without thoracotomy) and left cervical incision with hand-sewn anastomosis. Transthoracic oesophagectomy was performed by a separate right-sided thoracotomy and laparotomy or a left thoraco-abdominal approach with intra-thoracic anastomosis. A gastric tube was used as the conduit in all cases. The operations were carried out by one of three high-volume consultant surgeons at the centre during the study period. The choice of surgical approach did not vary between surgeons or over the years during the study period. Minimally invasive procedures were also conducted, but these were not included in the present study.

\section{Health-related quality of life (outcome)}

HRQOL was measured using well-established questionnaires, developed and validated by the European Organisation for Research and Treatment of Cancer (EORTC). ${ }^{18}$ The 30 -item cancer core questionnaire (EORTC QLQ-C30) has 9 multi-item scales, 1 measuring global quality of life, 5 measuring functions (physical-, role-, cognitive-, emotional- and social 
functioning), and 3 measuring symptoms (fatigue, pain, and nausea/vomiting), and also 6 single items measuring symptoms common among cancer patients in general (dyspnoea, appetite loss, insomnia, constipation, diarrhoea, and financial impact). ${ }^{18}$ Oesophageal cancer symptoms were measured with a specific module (EORTC QLQ-OG25) compromising 6 symptom scales (dysphagia, eating restrictions, reflux, odynophagia, pain and discomfort and anxiety) and 10 single items (eating in front of others, dry mouth, trouble with taste, body image, trouble swallowing saliva, choking when swallowing, trouble with coughing, trouble talking, weight loss, and hair loss). ${ }^{19}$ Each item (on both questionnaires) has a response on a four-point Likert-scale: 1) "not at all”, 2) "a little", 3) "quite a bit", and 4) "very much", except for the items in the global quality-of-life scale, which have seven response alternatives ranging from "very poor" to "excellent."

The baseline HRQOL questionnaires were gathered at admission for surgery (i.e. 6 weeks after the end of neoadjuvant therapy). These questionnaires were missing or eight of the patients $(5.5 \%)$ and questionnaires gathered at the initiation of neoadjuvant therapy were used instead of baseline questionnaires, because HRQOL after neoadjuvant therapy is known to recover before surgery. ${ }^{20,21}$ Subsequently, the follow-up questionnaires were collected at 6 months and 12 months after surgery.

\section{Statistical analysis}

The data management and statistical analyses were conducted by a senior biostatistician specialised in HRQOL analyses (AJ), who followed the analysis plan decided in a detailed and pre-defined study protocol. Chi-square test or Fisher's exact test were used to test potential differences in the baseline values between the exposure groups. HRQOL questionnaire responses were transformed into scores ranging from 0 to 100 . Higher scores correspond to better HRQOL in the function scales and the global quality of life scale, 
whereas higher scores in symptom scales and single items represent more problems. Missing responses were handled as recommended in the EORTC scoring manual. ${ }^{22}$ Missing scales and single items in the questionnaires, completely missing questionnaires, and missing clinical data were assumed to be missing at random and dealt with using complete case analysis. Mean scores for each surgical procedure were calculated with $95 \%$ confidence intervals (CI). Linear mixed-effects models were used to calculate mean score differences (MSD) with 95\% CIs between the two surgical approach groups. In all analyses, adjustments for potential confounding factors were made using all of the following a priori selected covariates that could influence both the surgical approach and HRQOL outcomes: baseline value for each HRQOL scale or individual item (continuous variable), age (continuous variable), ASA grade (categorised into 1-2 or 3-4), tumour location (categorised into oesophageal cancer or gastrooesophageal junction), tumour stage (categorised into 0-II or III), neoadjuvant chemotherapy (yes or no), adjuvant therapy started (yes or no) and complications (no complication, ClavienDindo I-II, or Clavien Dindo III-IV). Statistical significance was tested using the T-test only when the MSDs were at least 10 between groups, because such differences are considered clinically relevant and noticeable among patients according to previous studies, and such restrictive significance testing reduces the risk of chance errors. ${ }^{23,}{ }^{24}$ Power calculation showed that for $>80 \%$ power to detect a significant 2 -tailed p-value $(<0.05)$ for a clinically relevant difference of $\mathrm{MSD}=10$ with a standard deviation $(\mathrm{SD})$ of 11 , a sample size of at least 20 participants in each group would be required. The reference category in all statistical analyses was the transhiatal group. The statistical software SAS V.9.4 (SAS Institute, Cary, North Carolina, USA) was used for all analyses. 


\section{Results}

\section{Patients}

Among 251 patients who underwent oesophagectomy for oesophageal or gastro-oesophageal junction cancer at St. Thomas's Hospital during the study period, 228 patients underwent open transhiatal or transthoracic surgery and were thus eligible for the present study. Of these, $146(64.0 \%)$ patients with confirmed cancer in the resected specimen in the lower third of the oesophagus or gastro-oesophageal junction responded to the baseline HRQOL questionnaire and with follow-up until death or for at least 6 months at study termination were included in this study. Among these study participants, 86 (58.9\%) patients underwent transhiatal oesophagectomy and $60(41.1 \%)$ had transthoracic oesophagectomy. At the 6 month followup, $5(3.4 \%)$ patients had died, $21(14.4 \%)$ were too ill to answer the questionnaires (9 in the transhiatal and 12 in the transthoracic group), 2 (1.4\%) had missing questionnaires due to an administrative error and 7 (4.8\%) were non-responders for other reasons, leaving 111 patients (78.7\% of the 141 alive patients) for the final analysis. At the 12 month follow-up, 23 patients could not have been followed up long enough for the HRQOL assessment because of study termination. Of 123 patients with long enough follow-up, 20 (16.3\%) had died, 22 (17.9\%) were too ill to answer the questionnaires (11 in the transhiatal and 11 in the transthoracic group) and 7 (5.7\%) were non-responders for other reasons, leaving 74 patients $(71.8 \%$ of the alive patients) for final analysis. The non-participation rates among the alive patients were $16.7 \%$ for transhiatal and $28.1 \%$ for transthoracic oesophagectomy groups at 6 months, and $25.0 \%$ for transhiatal and $33.3 \%$ for transthoracic oesophagectomy groups at 12 months. The comparison between responders and non-responders showed that at 6 months there were no significant differences between the two groups (Supplementary table 1.), whereas at 12 months the non-responder group had proportionally more young patients and less females compared to the responders (Supplementary table 2). 
Characteristics of the study patients are presented in Table 1. Patients in the transhiatal oesophagectomy group were older, had more early-stage tumours and less often underwent neoadjuvant chemotherapy than those in the transthoracic group. No major difference in the occurrence of postoperative complications was observed (Table 1).

\section{Health-related quality of life 6 months after surgery}

Figure 1 and Supplementary Table 3 show the adjusted HRQOL mean scores from the general cancer questionnaire (QLQ-C30) at 6 months postoperatively. Compared to transhiatal oesophagectomy, transthoracic oesophagectomy was associated with clinically relevantly worse role function (MSD -12,95\% CI -23 to 0), which was also statistically significant ( $p=$ 0.046). Figure 1 and Supplementary Table 4 include the oesophageal cancer-specific issues. The transthoracic group had clinically relevantly more weight loss concerns (MSD 14, 95\% CI 0 to 27), but this difference was not statistically significant $(p=0.051)$.

\section{Health-related quality of life 12 months after surgery}

As shown in Figure 1 and Supplementary Table 5, at 12 months, the scores in the transthoracic group remained clinically relevantly worse for role function (MSD -10, 95\% CI -23 to 3), but this difference was not statistically significant $(p=0.121)$. Transthoracic group had clinically relevantly and statistically significantly worse nausea and vomiting (MSD 11, 95\% CI 0 to 22, $p=0.045$ ), dyspnoea (MSD 13, 95\% CI 1 to $25, p=0.029$ ), and constipation (MSD 20, 95\% CI 7 to 33, $p=0.003$ ) 12 months after surgery compared to the transhiatal group. Figure 1 and Supplementary Table 6 present the oesophageal cancer-specific concerns, and shows that the transthoracic group experienced clinically relevantly less oesophageal pain and discomfort (MSD -10, 95\% CI -22 to 1) compared to the transhiatal group, but the difference between groups was not statistically significant $(p=0.081)$. 


\section{Discussion}

This study suggests that transhiatal oesophagectomy is associated with clinically relevantly better HRQOL outcomes 6 and 12 months after surgery compared to transthoracic oesophagectomy. The role function at 6 months and nausea and vomiting, dyspnoea and constipation at 12 months were statistically significantly worse in the transthoracic group.

Among methodological strengths are the prospective and longitudinal study design with detailed clinical information, the use of well-validated HRQOL questionnaires, and the high rate of transhiatal (and transthoracic) procedures compared to most other centres. Surgical trials are prone to bias because of the individual surgeon's experience, but this was avoided by studying three high-volume surgeons with similar experience in both surgical approaches. A weakness is the limited sample size, which meant that some clinically relevant differences were not statistically significant despite the power calculation showing adequate statistical power for both time points. There were proportionally more patients in the transthoracic group that did not answer the HRQOL questionnaires because they were too ill, possibly biasing the results in favour of transthoracic approach as these patients would probably have scored the worst in the HRQOL assessments. The non-participation and loss to follow-up for other reasons than feeling too ill to answer the questionnaires could introduce selection bias, but it is unlikely that these factors were associated with the study exposure (surgical approach). However, there were no differences between responders and non-responders at 6 months follow-up in the non-respondent analysis, and minor differences in age- and sexdistribution between responders' and non-responders' at 12 months follow-up. Thus, any selection bias should be random, or in favour of the transthoracic surgery group. Confounding is a threat to observational studies in general. This bias by confounding was mitigated by adjusting for several potential confounding factors in the analyses. 
To the best of our knowledge, the only previous study comparing transthoracic and transhiatal oesophagectomy in relation to postoperative HRQOL was a Dutch randomised clinical trial, which showed similar results to the present study. ${ }^{14}$ Transhiatal oesophagectomy was associated with fewer physical symptoms and better activity levels compared to transthoracic oesophagectomy up to 3 months after surgery. Most of the HRQOL outcomes, such as physical, role and social functions, as well as pain and mental health scores slightly favoured transhiatal oesophagectomy in the Dutch study during the follow-up trajectory up to 18 months after surgery, but the differences between the groups were not statistically significant. ${ }^{14}$ It is important to also examine this research question in cohort studies because the inclusion criteria of cohort studies typically better mirror clinical practice better than randomised trials that often have strict inclusion criteria, which might exclude patients who have the worst HRQOL after surgery. ${ }^{25}$ It is important to note that neoadjuvant therapy was not used during the Dutch trial, ${ }^{14}$ which in turn might have affected the postoperative HRQOL in that study. ${ }^{20,21,26,27}$ The present study is therefore the first study to compare HRQOL between transhiatal and transthoracic oesophagectomy in the neoadjuvant treatment era.

The results of the present study suggest that several HRQOL measures are better after transhiatal oesophagectomy than transthoracic oesophagectomy. It is important to note that except for the major differences identified, most scales and items favoured the transhiatal approach, even though the MSDs were often between 5 and 10, and therefore of only a small clinically relevant level. Some of the differences could be related to the chest wall trauma and pain caused by thoracotomy, higher rates of respiratory complications and the greater sequelae of certain complications such as anastomotic leak in the mediastinum in the transthoracic oesophagectomy group, while leak following transhiatal oesophagectomy is 
located in the neck and therefore less serious. Thus, if thoracotomy is avoided, by using transhiatal oesophagectomy, it seems to improve postoperative HRQOL. The better scores measured in the nausea and vomiting, and constipation scales might speculatively reflect fewer gastric emptying-related and functional gastrointestinal problems after transhiatal oesophagectomy.

It would be valuable to confirm and validate the results of this study through studies of larger sample size, longer follow-up and from several centres. Moreover, it would be interesting to compare HRQOL outcomes between minimally invasive transhiatal or transthoracic approaches, and open transhiatal oesophagectomy. There are currently no registered ongoing trials on the topic.

In conclusion, several HROQL outcomes seem to be better after open transhiatal oesophagectomy compared to open transthoracic oesophagectomy for oesophageal cancer, particularly role function, nausea and vomiting, dyspnoea and constipation. Whilst decisions regarding the open surgical approach must be taken on an individual basis and include the consideration of various oncological factors and surgeons experience, these findings favour the use of transhiatal oesophagectomy in oesophageal cancer from a HRQOL perspective. 


\section{Acknowledgements}

We thank Sarah Stone and the Upper GI surgery team at St Thomas' Hospital for collecting study data. Funding was provided by Guy's and St Thomas' Charity, London, United Kingdom. The study sponsors had no role in the design of the study, data collection, analysis or interpretation of the results, the writing of the manuscript or the decision to submit the manuscript for publication. The data will not be made publicly available, but is available upon request from the authors. The research was not preregistered in an institutional registry. The authors' contributions to the study were as follows: conception and design, all authors; data collection, JAG, ARD, JZ, JL; data analysis, AJ; interpretation, all authors; drafting the manuscript, JHK; critical revision of the manuscript, all authors; study supervision, JL, PL; final approval, all authors.

\section{Conflict of Interest Statement}

The authors declare no potential conflicts of interest. 


\section{References}

1. Feith M, Stein HJ, Siewert JR. Adenocarcinoma of the esophagogastric junction: surgical therapy based on 1602 consecutive resected patients. Surg Oncol Clin N Am 2006;15(4): 751-764.

2. Vallbohmer D, Holscher AH, DeMeester S, DeMeester T, Salo J, Peters J, Lerut T, Swisher SG, Schroder W, Bollschweiler E, Hofstetter W. A multicenter study of survival after neoadjuvant radiotherapy/chemotherapy and esophagectomy for ypT0N0M0R0 esophageal cancer. Ann Surg 2010;252(5): 744-749.

3. Kauppila JH, Lagergren J. The surgical management of esophago-gastric junctional cancer. Surg Oncol 2016;25(4): 394-400.

4. Orringer MB, Sloan H. Esophagectomy without thoracotomy. J Thorac Cardiovasc Surg 1978;76(5): 643-654.

$5 . \quad$ Haverkamp L, Ruurda JP, van Leeuwen MS, Siersema PD, van Hillegersberg R. Systematic review of the surgical strategies of adenocarcinomas of the gastroesophageal junction. Surg Oncol 2014;23(4): 222-228.

6. Wei MT, Zhang YC, Deng XB, Yang TH, He YZ, Wang ZQ. Transthoracic vs transhiatal surgery for cancer of the esophagogastric junction: a meta-analysis. World J Gastroenterol 2014;20(29): 10183-10192.

$7 . \quad$ Boshier PR, Anderson O, Hanna GB. Transthoracic versus transhiatal esophagectomy for the treatment of esophagogastric cancer: a meta-analysis. Ann Surg 2011;254(6): 894-906.

8. Davies AR, Sandhu H, Pillai A, Sinha P, Mattsson F, Forshaw MJ, Gossage JA, Lagergren J, Allum WH, Mason RC. Surgical resection strategy and the influence of radicality on outcomes in oesophageal cancer. Br J Surg 2014;101(5): 511-517. 9. Jacobs M, Macefield RC, Elbers RG, Sitnikova K, Korfage IJ, Smets EM, Henselmans I, van Berge Henegouwen MI, de Haes JC, Blazeby JM, Sprangers MA. Metaanalysis shows clinically relevant and long-lasting deterioration in health-related quality of life after esophageal cancer surgery. Qual Life Res 2014;23(4): 1155-1176.

10. Djarv T, Metcalfe C, Avery KN, Lagergren P, Blazeby JM. Prognostic value of changes in health-related quality of life scores during curative treatment for esophagogastric cancer. J Clin Oncol 2010;28(10): 1666-1670.

11. Djarv T, Derogar M, Lagergren P. Influence of co-morbidity on long-term quality of life after oesophagectomy for cancer. Br J Surg 2014;101(5): 495-501.

12. Derogar M, Orsini N, Sadr-Azodi O, Lagergren P. Influence of major postoperative complications on health-related quality of life among long-term survivors of esophageal cancer surgery. J Clin Oncol 2012;30(14): 1615-1619.

13. Barreto JC, Posner MC. Transhiatal versus transthoracic esophagectomy for esophageal cancer. World J Gastroenterol 2010;16(30): 3804-3810.

14. de Boer AG, van Lanschot JJ, van Sandick JW, Hulscher JB, Stalmeier PF, de Haes JC, Tilanus HW, Obertop H, Sprangers MA. Quality of life after transhiatal compared with extended transthoracic resection for adenocarcinoma of the esophagus. J Clin Oncol 2004;22(20): 4202-4208.

15. Cunningham D, Allum WH, Stenning SP, Thompson JN, Van de Velde CJ, Nicolson M, Scarffe JH, Lofts FJ, Falk SJ, Iveson TJ, Smith DB, Langley RE, Verma M, Weeden S, Chua YJ, Participants MT. Perioperative chemotherapy versus surgery alone for resectable gastroesophageal cancer. N Engl J Med 2006;355(1): 11-20. 
16. Sobin LH, Gospodarowicz MK, Wittekind C, International Union against Cancer. TNM classification of malignant tumours (7th edn). Wiley-Blackwell: Chichester, West Sussex, UK ; Hoboken, NJ, 2010; xx, 309 p.

17. Dindo D, Demartines N, Clavien PA. Classification of surgical complications: a new proposal with evaluation in a cohort of 6336 patients and results of a survey. Ann Surg 2004;240(2): 205-213.

18. Aaronson NK, Ahmedzai S, Bergman B, Bullinger M, Cull A, Duez NJ, Filiberti A, Flechtner H, Fleishman SB, de Haes JC, et al. The European Organization for Research and Treatment of Cancer QLQ-C30: a quality-of-life instrument for use in international clinical trials in oncology. J Natl Cancer Inst 1993;85(5): 365-376.

19. Lagergren P, Fayers P, Conroy T, Stein HJ, Sezer O, Hardwick R, Hammerlid E, Bottomley A, Van Cutsem E, Blazeby JM, European Organisation for Research Treatment of Cancer G, Quality of Life G. Clinical and psychometric validation of a questionnaire module, the EORTC QLQ-OG25, to assess health-related quality of life in patients with cancer of the oesophagus, the oesophago-gastric junction and the stomach. Eur J Cancer 2007;43(14): 2066-2073.

20. Blazeby JM, Sanford E, Falk SJ, Alderson D, Donovan JL. Health-related quality of life during neoadjuvant treatment and surgery for localized esophageal carcinoma. Cancer 2005;103(9): 1791-1799.

21. Safieddine N, Xu W, Quadri SM, Knox JJ, Hornby J, Sulman J, Wong R, Guindi M, Keshavjee S, Darling G. Health-related quality of life in esophageal cancer: effect of neoadjuvant chemoradiotherapy followed by surgical intervention. J Thorac Cardiovasc Surg 2009;137(1): 36-42.

22. Fayers P, Aaronson N, Bjordal K, Groenvold M, Curran D, Bottomley A. The EORTC QLQ-C30 Scoring Manual ( 3rd Edition edn). European Organisation for Research and Treatment of Cancer: Brussels, 2001.

23. Osoba D, Rodrigues G, Myles J, Zee B, Pater J. Interpreting the significance of changes in health-related quality-of-life scores. J Clin Oncol 1998;16(1): 139-144.

24. King MT. The interpretation of scores from the EORTC quality of life questionnaire QLQ-C30. Qual Life Res 1996;5(6): 555-567.

$25 . \quad$ Booth CM, Tannock IF. Randomised controlled trials and population-based observational research: partners in the evolution of medical evidence. Br J Cancer 2014;110(3): 551-555.

26. Hauser C, Patett C, von Schoenfels W, Heits N, Schafmayer C, Malchow B, Hampe J, Schniewind B, Becker T, Egberts JH. Does neoadjuvant treatment before oncologic esophagectomy affect the postoperative quality of life? A prospective, longitudinal outcome study. Dis Esophagus 2015;28(7): 652-659.

27. Reynolds JV, McLaughlin R, Moore J, Rowley S, Ravi N, Byrne PJ. Prospective evaluation of quality of life in patients with localized oesophageal cancer treated by multimodality therapy or surgery alone. Br J Surg 2006;93(9): 1084-1090. 
Table 1. Characteristics of 146 study patients who underwent open transhiatal or transthoracic oesophagectomy for oesophageal cancer.

\begin{tabular}{|c|c|c|c|c|}
\hline 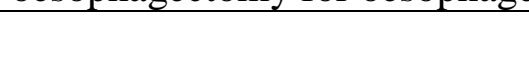 & Transhiatal & Transthoracic & Total & $p$-value \\
\hline & Number (\%) & Number (\%) & Number (\%) & Transhiatal \\
\hline \multirow[t]{2}{*}{ Total } & $86(58.9)$ & $60(41.1)$ & $146(100.0)$ & vs. \\
\hline & & & & Transthoracic \\
\hline \multicolumn{5}{|l|}{ Age (in years) } \\
\hline$\leq 66$ & $38(44.2)$ & $38(63.3)$ & $76(52.1)$ & $p=0.023$ \\
\hline$>67$ & $48(55.8)$ & $22(36.7)$ & $70(48.0)$ & \\
\hline \multicolumn{5}{|l|}{ Sex } \\
\hline Male & $66(76.7)$ & $52(86.7)$ & $118(80.8)$ & $p=0.134$ \\
\hline Female & $20(23.3)$ & $8(13.3)$ & $28(19.2)$ & \\
\hline \multicolumn{5}{|l|}{ Cohabitation status } \\
\hline Living alone & $15(17.4)$ & $13(21.7)$ & $28(19.2)$ & $p=0.041^{\mathrm{a}}$ \\
\hline Cohabiting & $63(73.3)$ & $47(78.3)$ & $110(75.3)$ & \\
\hline Missing & $8(9.3)$ & $0(0.0)$ & $8(5.5)$ & \\
\hline \multicolumn{5}{|l|}{ Work status } \\
\hline Working & $29(33.7)$ & $30(50.0)$ & $59(40.4)$ & $p=0.049$ \\
\hline Not working & $57(66.3)$ & $30(50.0)$ & $87(59.6)$ & \\
\hline \multicolumn{5}{|l|}{ ASA-grade* } \\
\hline $1-2$ & $60(69.8)$ & $41(68.3)$ & $101(69.2)$ & $p=0.854$ \\
\hline $3-4$ & $26(30.2)$ & $19(31.7)$ & $45(30.8)$ & \\
\hline \multicolumn{5}{|l|}{ Tumour location } \\
\hline Lower third & $47(54.7)$ & $29(48.3)$ & $76(52.1)$ & $p=0.452$ \\
\hline Junctional & $39(45.3)$ & $31(51.7)$ & $70(48.0)$ & \\
\hline \multicolumn{5}{|l|}{ Tumour stage } \\
\hline $0-\mathrm{I}$ & $13(15.1)$ & $3(5.0)$ & $16(11.0)$ & $p=0.015$ \\
\hline II & $22(25.6)$ & $8(13.3)$ & $30(20.6)$ & \\
\hline III & $51(59.3)$ & $49(81.7)$ & $100(68.5)$ & \\
\hline \multicolumn{5}{|l|}{ Neoadjuvant therapy } \\
\hline Yes & $70(81.4)$ & $58(96.7)$ & $128(87.7)$ & $p=0.006$ \\
\hline No & $16(18.6)$ & $2(3.3)$ & $18(12.3)$ & \\
\hline \multicolumn{5}{|l|}{ Adjuvant therapy } \\
\hline Not recommended & $18(20.9)$ & $6(10.0)$ & $24(16.4)$ & $p=0.154$ \\
\hline Recommended, not started & $19(57.0)$ & $12(20.0)$ & $31(21.2)$ & \\
\hline Recommended, started & $49(22.1)$ & $41(68.3)$ & $90(61.6)$ & \\
\hline Missing & $0(0.0)$ & $1(1.7)$ & $1(0.6)$ & \\
\hline \multicolumn{5}{|l|}{$\begin{array}{l}\text { Clavien-Dindo Classification } \\
\text { of Complications }\end{array}$} \\
\hline None & $39(45.4)$ & $28(46.7)$ & $67(45.9)$ & $p=0.614$ \\
\hline
\end{tabular}




\begin{tabular}{|l|c|c|c|c|}
\hline Grades 1-2 & $32(37.2)$ & $25(41.7)$ & $57(39.0)$ & \\
\hline Grades 3-4 & $15(17.4)$ & $7(11.7)$ & $22(15.1)$ & \\
\hline
\end{tabular}

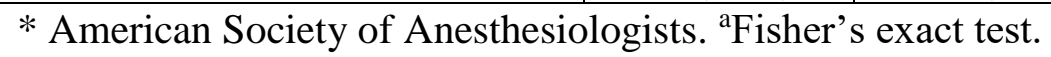




\section{Figure Legends:}

Figure 1. Adjusted health-related quality-of-life (HRQoL) mean score differences on the European Organization for Research and Treatment of Cancer QLQ-C30 (A functional scales, B symptoms scales and items) and QLQ-OG25 (C symptoms scales, D single items) between patients having undergone open transhiatal or transthoracic oesophagectomy at 6 months (blue line) and 12 months of follow-up (red line). Scores below 0 favour transhiatal oesophagectomy in A, and scores above 0 favour transhiatal oesophagectomy in B, C and D. 